\title{
Tests of Lorentz invariance using hydrogen molecules
}

\author{
Holger Müller* Sven Herrmann, Alejandro Saenz, and Achim Peters \\ Institut für Physik, Humboldt-Universität zu Berlin, Hausvogteiplatz 5-7, 10117 Berlin, Germany. \\ Tel. +49 (30) 2093-4907, Fax +49 (30) 2093-471 \\ Claus Lämmerzahl \\ ZARM (Center for Applied Space Technology and Microgravity), \\ Universität Bremen, Am Fallturm, 28359 Bremen, Germany
}

(Dated:)

\begin{abstract}
We discuss the consequences of Lorentz violation (as expressed within the Lorentz-violating extension of the standard model) for the hydrogen molecule, which represents a generic model of a molecular binding. Lorentz-violating shifts of electronic, vibrational and rotational energy levels, and of the internuclear distance are calculated. This offers the possibility of obtaining improved bounds on Lorentz invariance by experiments using molecules.

PACS numbers: 11.30.Cp, 03.30.+p, 04.80.Cc, 03.50.De
\end{abstract}

\section{INTRODUCTION}

A violation of Lorentz invariance occurs in many current models of quantum gravity. While such theories typically operate on the Planck energy scale, low-energy remnants of Planck scale physics might break Lorentz invariance in the equations of motion of some or all of the particles of the standard model. Such Lorentz violation is generally described by the standard model extension (SME) 1]. Currently, there is a large experimental effort to find sharp limits on Lorentz violation for the different sectors of the standard model, and many types of Lorentz violation for different particles have been constrained 2, 3].

Here, we consider the quantum electrodynamical sector of the SME that includes photons and electrons. Stringent limits on Lorentz violation in the other sectors allow us to neglect the possibility of Lorentz violation in other sectors for this work. For photons, atoms (e.g., also for atomic hydrogen [4]), and many sub-atomic particles, consequences of Lorentz violation have been studied extensively, see [2, 3, 4, 5] and references therein. However, many high-precision experiments are performed on chemically bonded systems like molecules (e.g., high resolution spectroscopy) or solids (e.g. experiments with macroscopic cavity resonators $6,6,8,9]$ ). A study of the influence of Lorentz violation on chemical bonds is thus interesting.

As a generic model of chemical bonds, we investigate neutral and ionized molecular hydrogen $\mathrm{H}_{2}$ and $\mathrm{H}_{2}^{+}$under the influence of Lorentz violation in the electrons' equation of motion. These molecules are simple enough so that a specific wave-function can be used as an ansatz for

\footnotetext{
*Now at Physics Department, Varian Bldg., Room 226, Stanford University, Stanford, CA 94305-4060, Phone: (650) 725-2354, Fax: (650) 723-9173; Electronic address: holgerm@stanford.edu

${ }^{\dagger}$ Electronic address: achim.peters@physik.hu-berlin.de
}

the calculations. At the same time, they exhibit interesting features not found in simpler systems (like hydrogen atoms): For example, a preferred direction is singled out by the molecules' axis; the internuclear distance defines a length standard whose behavior in the case of Lorentz violation can explicitly be studied (and compared to other different length standards, like crystals [10, 11] or the distance traveled by a ray of light within a certain time).

Experiments aiming for sympathetic cooling (to $\mathrm{mK}$ temperature) and high-resolution spectroscopy of $\mathrm{H}_{2}^{+}$and $\mathrm{HD}^{+}$are currently under way [12, 13]. Using the theory presented here, limits on Lorentz violation can be obtained in these experiments by searching for a Lorentzviolating shift in the transition frequencies. With the high resolution that is possible in frequency metrology and the suppression of line broadening mechanisms for the cooled molecules, such experiments may improve the present bounds on particular Lorentz violating parameters for the electron.

We treat the hydrogen molecule using the BornOppenheimer model that is described in textbooks, e.g. 14]. The motion of the nuclei is neglected and approximate electron wave functions are obtained by linear combination of atomic orbitals. The Born-Oppenheimer model is a basic model with sufficient precision for our purposes, as we do not need to predict the absolute values of the quantities, but only the Lorentz violating shifts of them. We work to first order in the Lorentz violating parameters throughout. Since the nuclei are assumed to be point-like, our results are valid for $\mathrm{H}_{2}$ and $\mathrm{H}_{2}^{+}$as well as for $\mathrm{HD}$ and $\mathrm{HD}^{+}$.

In Sec. III we calculate the matrix elements of the Lorentz violating quantities from the SME for $\mathrm{H}_{2}$ and $\mathrm{H}_{2}^{+}$, taking into account that usually the molecules will be in an angular momentum eigenstate. In Sec. III we calculate the modified ground state energy, bond length, and frequencies of rotational and vibrational transitions. In Sec. [V] we discuss the possibility of improving present limits on Lorentz violation by experiments using molecules. The appendix gives the explicit time- 
dependence of the hypothetical signal for Lorentz violation.

\section{Standard model extension (SME)}

The SME 1, 2, 3, 4] starts from a Lagrangian formulation of the standard model, adding all possible observer Lorentz scalars that can be formed from the known particles and Lorentz tensors. The non-relativistic Schrödinger Hamiltonian $h=\hat{h}+\delta h$ of a free electron derived from the SME Lagrangian is the sum of the usual free-particle Hamiltonian $\hat{h}$ and a Lorentz-violating term. Disregarding a constant term that has no physical consequences and terms proportional to odd powers of the particle momentum that vanish in the center of mass system [1, 3, 15, 16],

$$
\delta h=m_{e} c^{2} B_{j}^{\prime} \sigma^{j}+E_{j k} \frac{p_{j} p_{k}}{m_{e}}+F_{j k l} \frac{p_{j} p_{k}}{m_{e}} \sigma^{l} .
$$

Here, $p_{j}(j=1,2,3)$ are the components of the 3 momentum, $\sigma^{j}$ are the Pauli matrices, and $m$ is the mass of the electron. The abbreviations

$$
\begin{aligned}
B_{j}^{\prime}= & -\frac{b_{j}}{m}+d_{j 0}-\frac{1}{2} \varepsilon_{j k l} g_{k l 0}+\frac{1}{2 m} \varepsilon_{j k l} H_{k l} \\
E_{j k}^{\prime}= & -c_{j k}-\frac{1}{2} c_{00} \delta_{j k} \\
F_{j k l}^{\prime}= & {\left[\left(d_{0 j}+d_{j 0}\right)-\frac{1}{2}\left(\frac{b_{j}}{m}+d_{j 0}+\frac{1}{2} \varepsilon_{j m n} g_{m n 0}\right.\right.} \\
& \left.\left.+\frac{1}{2 m} \varepsilon_{j m n} H_{m n}\right)\right] \delta_{k l}+\frac{1}{2}\left(\frac{b_{l}}{m}+\frac{1}{2} \varepsilon_{l m n} g_{m n 0}\right) \delta_{j k} \\
& -\varepsilon_{j l m}\left(g_{m 0 k}+g_{m k 0}\right)
\end{aligned}
$$

contain the Lorentz tensors $b_{\mu}, c_{\mu \nu}, d_{\mu \nu}, g_{\lambda \mu \nu}$, and $H_{\mu \nu}$ that encode Lorentz violation for the fermions within the SME defined in 1, 2]. In this work, we deal with electrons, so all these parameters are electron parameters. For neutral hydrogen $\mathrm{H}_{2}$, this perturbation has to be summed over the two electrons. To first order in the changes, the influence of the Lorentz violating perturbation is given by its matrix elements.

\section{MOLECULAR HYDROGEN}

We will first assume non-rotating molecules; the rotation of the molecules will be treated subsequently. Practically, the influence of the rotation on the other properties of the molecule is small, so we may neglect it here.

\section{A. Neutral hydrogen molecule}

We are using atomic units $m=\hbar=e=1$, where $e$ is the electron's charge. We denote the two nuclei $a$ and $b$, and enumerate the electrons 1,2 . Let $\left(x_{1}\right)_{j}$ and $\left(x_{2}\right)_{j}$ be

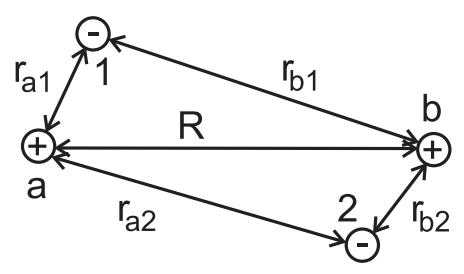

FIG. 1: Definition of coordinates. $a$ and $b$ denote the nuclei, 1 and 2 the electrons.

the spatial components of the position of electron 1 and 2 , respectively, and $\left(p_{1}\right)_{j},\left(p_{2}\right)_{j}$ those of their momenta. Let $\vec{r}_{a 1}$ be the distance between nucleus $a$ and electron 1 , and so forth, and $\vec{R}$ the distance between the nuclei (see Fig. 11). For this calculation, we adopt coordinates such that $\vec{R}$ is parallel to the $z$ axis.

The antisymmetric wave function of $\mathrm{H}_{2}$ can be written as the product of a spatial function $\Psi_{r}$ and a spin function that is antisymmetric under exchange of the electrons. Thus, the matrix elements of the spin-dependent terms of $\delta h$ vanish and the $\mathrm{H}_{2}$ molecule will only be sensitive to the Lorentz violating quantity $E_{j k}$ of Eq. (1).

We make the usual ansatz for the spatial wave function of $\mathrm{H}_{2}$ 14]:

$$
\Psi_{r}=\alpha\left[f\left(\vec{r}_{a 1}\right) f\left(\vec{r}_{b 2}\right)+f\left(\vec{r}_{b 1}\right) f\left(\vec{r}_{a 2}\right)\right]
$$

with a normalization factor $\alpha=1 / \sqrt{2\left(1+S^{2}\right)}$, where

$$
S=\int f^{*}\left(\vec{r}_{a 1}\right) f\left(\vec{r}_{b 1}\right) d^{3} x_{1}=\left[1+\gamma R+\frac{1}{3}(\gamma R)^{2}\right] e^{-\gamma R}
$$

is the overlap integral. The function

$$
f(\vec{r})=\sqrt{\frac{\gamma^{3}}{\pi}} e^{-\gamma r}
$$

satisfies $\int|f(\vec{r})|^{2} d^{3} r=1$. For $\gamma=1$, it is the usual single-electron wave function of the hydrogen atom in the ground state. The ground state of $\mathrm{H}_{2}$ is found by minimizing the energy $U_{\mathrm{H}_{2}}$ of the system as a function of $R$ and $\gamma$. For $\mathrm{H}_{2}$, the global minimum is obtained at $R_{0}=1.41$ with $\gamma=1.166: U_{\mathrm{H}_{2}}\left(R_{0}\right)=-1.139$ [14, 17]. For each $R$, there is a $\gamma(R)$ that gives a local minimum for $U_{\mathrm{H}_{2}}$. When calculating the dependence of $U_{\mathrm{H}_{2}}$ on $R$, the dependence of $\gamma(R)$ has to be taken into account, since the electronic wave functions will adjust themselves for each $R$ rapidly compared to, e.g., the molecular vibrations.

The Lorentz violating changes are induced by the matrix elements $\left\langle p_{i} p_{j}\right\rangle$. Due to symmetry under inversion of the $x$ and $y$ axes (that also holds for $\mathrm{HD}$ and $\mathrm{HD}^{+}$), $\left\langle p_{i} p_{j}\right\rangle=0$ unless $i=j$ (which can also be verified by explicit calculation in analogy to Eq. (12)). Furthermore $\left\langle p_{x}^{2}\right\rangle=\left\langle p_{y}^{2}\right\rangle$. Thus,

$$
\langle\delta h\rangle_{\mathrm{H}_{2}}=E_{i i}\left\langle p_{i} p_{i}\right\rangle_{\mathrm{H}_{2}}
$$


with

$$
\begin{aligned}
\left\langle p_{i} p_{i}\right\rangle_{\mathrm{H}_{2}} & =-\int \Psi_{r}^{*}\left[\left(\partial_{i}^{2}\right)_{1}+\left(\partial_{i}^{2}\right)_{2}\right] \Psi_{r} d^{3}(x)_{1} d^{3}(x)_{2} \\
& =-4 \alpha^{2}\left[\mathcal{A}_{i}^{\prime}+S \mathcal{A}_{i}^{\prime \prime}\right]
\end{aligned}
$$

where we denoted $\left(\partial_{j}^{2}\right)_{1} \equiv \partial^{2} /\left(\partial\left(x_{1}\right)^{2}\right)$, and

$$
\begin{aligned}
& \mathcal{A}_{j}^{\prime}=\int f^{*}\left(\vec{r}_{a 1}\right) \frac{\partial^{2}}{\partial\left(x_{1}\right)_{j}^{2}} f\left(\vec{r}_{a 1}\right) d^{3}(x)_{1}=-\frac{\gamma^{2}}{3}, \\
& \mathcal{A}_{j}^{\prime \prime}=\int f\left(\vec{r}_{b 1}\right) \frac{\partial^{2}}{\partial\left(x_{1}\right)_{j}^{2}} f^{*}\left(\vec{r}_{a 1}\right) d^{3}(x)_{1} .
\end{aligned}
$$

The first integral is evaluated using elementary integrations in polar coordinates with the $\theta=0$ direction parallel to $x_{j}$. For $\mathcal{A}_{j}^{\prime \prime}$, we insert Eq. (7):

$\mathcal{A}_{j}^{\prime \prime}=-\frac{\gamma^{4}}{\pi} \int e^{-\gamma\left(r_{a 1}+r_{b 1}\right)}\left(\frac{1}{r_{a 1}}-\frac{\left(x_{j}\right)_{1}^{2}}{r_{a 1}^{3}}-\gamma \frac{\left(x_{j}\right)_{1}^{2}}{r_{a 1}^{2}}\right) d^{3} x_{1}$

We now use polar coordinates $(r, \theta, \phi)$ with nucleus $a$ as center and the $\theta=0$ axis pointing towards nucleus $b$ :

$\mathcal{A}_{j}^{\prime \prime}=\frac{\gamma^{4}}{\pi} \int e^{-\gamma\left(r+r_{b 1}\right)}\left(\frac{\left(x^{j}\right)_{1}^{2}}{r}+\gamma\left(x^{j}\right)_{1}^{2}-r\right) \sin \theta d r d \theta d \phi$

with

$$
\begin{aligned}
x^{1} \equiv x & =r \cos \theta \\
x^{2} \equiv y & =r \sin \theta \sin \phi \\
x^{3} \equiv z & =r \sin \theta \cos \phi \\
r_{b 1}^{2} & =r^{2}-z^{2}+(z+R)^{2} .
\end{aligned}
$$

Numerical computation yields the values for $\left\langle p_{x} p_{x}\right\rangle_{\mathrm{H}_{2}}=$ $\left\langle p_{y} p_{y}\right\rangle_{\mathrm{H}_{2}},\left\langle p_{z} p_{z}\right\rangle_{\mathrm{H}_{2}}$ and their derivatives $d^{n}\left\langle p_{i} p_{i}\right\rangle_{\mathrm{H}_{2}} / d R^{n}$ given in Tab. 【

\section{B. Ionized hydrogen molecule}

Ionized hydrogen $\mathrm{H}_{2}^{+}$can be treated analogously. However, since there is only one electron, the spin dependent terms do not vanish, and we have to deal with the full Lorentz violating correction of the Hamiltonian, Eq. (1). The wave function for $\mathrm{H}_{2}$ is modeled as

$$
\psi=\beta\left[f\left(\vec{r}_{a}\right)+f\left(\vec{r}_{b}\right)\right]
$$

with a normalization factor $\beta=1 / \sqrt{1+S}$ and $f$ as defined in Eq. (7). For $\mathrm{H}_{2}^{+}, \gamma=1.24$ and $R_{0}=2.00$ give minimum total energy [14]. Again, $\gamma=\gamma(R)$ has to be taken into account. We can write the Lorentz violating contribution as

$$
\langle\delta h\rangle_{\mathrm{H}_{2}^{+}}=B_{i} \sigma^{i}-\tilde{E}_{i i}\left\langle p_{i} p_{i}\right\rangle_{\mathrm{H}_{2}^{+}}
$$

(again, $\left\langle p_{i} p_{j}\right\rangle=0$ for $i \neq j$ ) with

$$
\tilde{E}_{j k}=E_{j k}+F_{j k l} \sigma_{l} .
$$

TABLE I: $\left\langle p_{x} p_{x}\right\rangle=\left\langle p_{y} p_{y}\right\rangle,\left\langle p_{z} p_{z}\right\rangle$ and their derivatives $\left(d^{n}\left\langle p_{i} p_{i}\right\rangle / d R^{n}\right)_{R_{0}}$ for $\mathrm{H}_{2}\left(\right.$ at $\left.R_{0}=1.414\right)$ and $\mathrm{H}_{2}^{+}\left(R_{0}=\right.$ 2.00). Also shown is the unperturbed energy $U(R)$ and its derivatives $\left(d^{n} U / d R^{n}\right)_{R_{0}}$. All these are total derivatives, with $\gamma(R)$ (also tabulated together with its derivatives) also depending on $R$. The first derivative $(d U / d R)_{R_{0}}$ vanishes, since $R_{0}$ minimizes $U$.

\begin{tabular}{ccccc}
\hline$n$ & 0 & 1 & 2 & 3 \\
\hline$\left\langle p_{x} p_{x}\right\rangle_{\mathrm{H}_{2}}$ & 0.833 & -0.344 & 0.454 & \\
$\left\langle p_{z} p_{z}\right\rangle_{\mathrm{H}_{2}}$ & 0.612 & -0.266 & 0.501 & \\
$\gamma_{\mathrm{H}_{2}}$ & 1.166 & -0.238 & 0.233 & -0.150 \\
$U_{\mathrm{H}_{2}}$ & -1.139 & 0.000 & 0.374 & -0.849 \\
$\left\langle p_{x} p_{x}\right\rangle_{\mathrm{H}_{2}^{+}}$ & 0.451 & -0.147 & 0.147 & \\
$\left\langle p_{z} p_{z}\right\rangle_{\mathrm{H}_{2}^{+}}$ & 0.271 & -0.084 & 0.134 & \\
$\gamma_{\mathrm{H}_{2}^{+}}$ & 1.238 & -0.203 & 0.150 & -0.112 \\
$U_{\mathrm{H}_{2}^{+}}$ & -0.587 & 0.000 & 0.100 & -0.259 \\
\hline
\end{tabular}

and

$$
\left\langle p_{j} p_{j}\right\rangle_{\mathrm{H}_{2}^{+}}=\int \psi^{*} \frac{\partial^{2}}{\partial x_{j}^{2}} \psi d^{3} x=-\beta\left(\mathcal{A}_{j}^{\prime}+\mathcal{A}_{j}^{\prime \prime}\right) .
$$

With $\mathcal{A}_{j}^{\prime}, \mathcal{A}_{j}^{\prime \prime}$ as above. The results are given in Tab. 【

\section{Rotation of the molecules}

As shown above, the expectation value of the Lorentz violation for neutral and ionized hydrogen depend only on two parameter combinations that we may choose as the trace $\operatorname{tr}(E)$ and $E_{3}$,

$$
\begin{aligned}
\operatorname{tr}(E) & =E_{x x}+E_{y y}+E_{z z}, \\
E_{3} & =E_{x x}+E_{y y}-2 E_{z z}=\operatorname{tr}(E)-3 E_{z z},
\end{aligned}
$$

and similar for $\tilde{E}$. Spatial rotations, given by the matrix

$$
R(\theta, \phi)=\left(\begin{array}{ccc}
\cos \phi & \cos \theta \sin \phi & \sin \phi \sin \theta \\
-\sin \phi & \cos \phi \cos \theta & \cos \phi \sin \theta \\
0 & -\sin \theta & \cos \theta
\end{array}\right),
$$

will leave $\operatorname{tr}(E)$ unchanged, while $E_{z z} \rightarrow E_{z z}(\theta, \phi)$ with

$$
\begin{aligned}
E_{z z}(\theta, \phi) & =\sin ^{2} \theta\left[E_{y y} \cos ^{2} \phi+E_{x x} \sin ^{2} \phi+E_{x y} \sin 2 \phi\right] \\
& +\sin 2 \theta\left[E_{y z} \cos \phi+E_{x z} \sin \phi\right]+E_{z z} \cos ^{2} \theta,
\end{aligned}
$$

which leads to a change $E_{3} \rightarrow E_{3}(\theta, \phi)$.

Now we consider molecules whose axes are not fixed: Due to rotation invariance without Lorentz violation, the wave function of the unperturbed molecules can be written as the product of the above wave functions and spherical harmonics $Y_{l}^{m}(\theta, \phi)$. Thus, $\delta h$ has to be averaged with the angular wave functions $|l m\rangle$. Due to the rotation invariance of $\operatorname{tr}(E),\langle l m|\operatorname{tr}(E)| l m\rangle=\operatorname{tr}(E)$. For 
$\left(E_{3}\right)_{l}^{m}:=\left\langle l m\left|E_{3}\right| l m\right\rangle$, we express the sine and cosine functions in Eq. (20) in terms of spherical harmonics:

$$
\begin{gathered}
E_{3}(\theta, \phi)=-\frac{1}{3} \operatorname{tr}(E)+E_{3} \sqrt{\frac{4 \pi}{5}} Y_{2}^{0}+E_{x x} X_{2}^{2+} \\
+E_{y y} X_{2}^{2+}+2 i E_{x y} X_{2}^{2-}+2 E_{y z} X_{2}^{1-}-2 i E_{x z} X_{2}^{1+}
\end{gathered}
$$

where we used the abbreviation

$$
X_{l}^{m \pm}=\sqrt{\frac{6 \pi}{5}}\left(Y_{l}^{m} \pm Y_{l}^{-m}\right) .
$$

The average is calculated by an application of the Wigner-Eckart theorem [18]. $\left\langle\operatorname{lm}\left|Y_{l}^{m}\right| l m\right\rangle=0$ unless $m=0$, which removes all but the first two terms of Eq. (21). After some algebra, we find

$$
\begin{aligned}
\left(E_{3}\right)_{l}^{m} & =-(-1)^{m}(2 l+1)\left(\begin{array}{ccc}
l & l & 2 \\
0 & 0 & 0
\end{array}\right)\left(\begin{array}{ccc}
l & l & 2 \\
m & -m & 0
\end{array}\right) E_{3} \\
& =2 \frac{(l+1)\left[3 m^{2}-l(l+1)\right]}{(2 l-1)(2 l+2)(2 l+3)} E_{3}
\end{aligned}
$$

and analogous for $\tilde{E}_{z z}$. The brackets are the $3 j$-symbols 18 .

The situation that the quantization axis itself is rotated (e.g. by fixing it in the lab and using Earth's rotation) can be most easily treated by transforming the Lorentz-violating SME tensor $c_{\mu \nu}$ into a co-rotating frame, as dicussed in the appendix. (The same situation can be described by a time-dependent superposition of the eigenfunctions $Y_{l}^{m}$ for a fixed quantization axis. Some elements of $E_{i j}$ would enter the expectation value in terms of the form $\left\langle l_{1} m_{l}\left|Y_{l}^{m}\right| l_{2} m_{2}\right\rangle$, which is nonzero for $m_{2}-m_{1}+m=0$.)

\section{MODIFIED PROPERTIES OF THE GROUND STATES}

\section{A. Energy}

The energy $U$ of the ground state is shifted by the expectation value $\langle\delta h\rangle$. From the numerical values given in table【 we obtain for $\mathrm{H}_{2}$ and $H_{2}^{+}$, respectively [19]

$$
\begin{aligned}
& \left(\frac{\delta U}{U}\right)_{\mathrm{H}_{2}}=-0.667 \operatorname{tr}(E)-0.065\left(E_{3}\right)_{l}^{m} \\
& \left(\frac{\delta U}{U}\right)_{\mathrm{H}_{2}^{+}}=-0.667 \operatorname{tr}(\tilde{E})-0.102\left(\tilde{E}_{3}\right)_{l}^{m}+\frac{m_{e} c^{2}}{U_{\mathrm{H}_{2}^{+}}} B_{i} \sigma^{\dot{\psi}}(24)
\end{aligned}
$$

Here, the molecules are assumed to be in an eigenstate of the angular momentum with quantum numbers $l$ and $m$. For molecules with a fixed orientation, $\left(E_{3}\right)_{l}^{m}$ is to be replaced by $E_{3}$. For $\mathrm{H}_{2}$ in the ground state, the spindependent term proportional to $m_{e} c^{2} / U_{\mathrm{H}_{2}^{+}} \simeq 3.2 \times 10^{4}$ drops out, as the spins of the electrons are antiparallel.
Precision ab initio calculations 20 and measurements 21,22 of the ground state energy of $\mathrm{H}_{2}$ agree to an impressive precision of $\delta U / U \lesssim 1 \times 10^{-7}$. According to Eq. (24), this gives an upper limit of $|\operatorname{tr}(E)| \lesssim 1 \times 10^{-7}$ and $E_{3} \lesssim 10^{-6}$ (as molecules with many different orientations of the quantization axis and combinations of $l, m$ enter the measurements). However, more precise limits were already derived 11] from electromagnetic cavity experiments [6, 7], as discussed below.

\section{B. Bond length}

The change of the bond length $R_{0}$ can be calculated by minimizing the total energy

$$
U=U_{0}+\left\langle(\delta h)_{\mathrm{H}_{2}}\right\rangle
$$

of the molecule. $U_{0}$ is the energy without Lorentz violating terms, as it is calculated in the literature. It has a minimum at $R_{0}$, the bond length of $\mathrm{H}_{2}$. With Lorentz violating terms,

$$
U\left(R_{0}+\delta R\right)=\text { const. }+\frac{1}{2} \frac{\partial^{2} U_{0}}{\partial R^{2}}(\delta R)^{2}+\frac{\partial\langle(\delta h)\rangle}{\partial R} \delta R,
$$

so the new energy minimum will be at a modified length $R=R_{0}+(\delta R)_{\min }$. Setting $\partial U(R) / \partial R=0$ and inserting the numerical values from Tab. I leads to

$$
\begin{aligned}
& \left(\frac{\delta R}{R_{0}}\right)_{\mathrm{H}_{2}}=0.603 \operatorname{tr}(E)+0.050\left(E_{3}\right)_{l}^{m}, \\
& \left(\frac{\delta R}{R_{0}}\right)_{\mathrm{H}_{2}^{+}}=0.632 \operatorname{tr}(\tilde{E})+0.106\left(\tilde{E}_{3}\right)_{l}^{m} .
\end{aligned}
$$

The term proportional to $B_{j}$ does not contribute here and below, as it does not depend on the internuclear distance $R$. As the bond length has not been measured to a similar precision as energy levels, a comparison between theory and experiment does presently not lead to interesting limits on Lorentz violation.

\section{Vibrational transitions}

The energy levels of a quantized harmonic oscillator $U_{v}=(v+1 / 2) \hbar \omega$, where $\omega$ is the resonance frequency and $v=1,2, \ldots$ the vibrational quantum number, are equidistant with a difference $\hbar \omega$ between neighbors. For the hydrogen molecule, $\omega=\sqrt{k / \bar{m}}$, where $k=\partial^{2} U / \partial R^{2}$ and $\bar{m}=m_{a} m_{b} /\left(m_{a}+m_{b}\right)$ is the reduced mass of the nuclei. For the change of $\partial^{2} U / \partial R^{2}$, we take into account both the addition of $\delta h$ as well as the change due to the shift $\delta R$ in the internuclear distance:

$$
\left(\frac{\partial^{2} U}{\partial R^{2}}\right)_{R_{0}+\delta R}=\left(\frac{\partial^{2} U}{\partial R^{2}}\right)_{R_{0}}+\left(\frac{\partial\langle\delta h\rangle}{\partial R^{2}}\right)_{R_{0}}+\left(\frac{\partial^{3} U}{\partial R^{3}}\right)_{R_{0}} \delta R .
$$


(We can use $\langle\delta h\rangle\left(R_{0}+\delta R\right) \approx\langle\delta h\rangle\left(R_{0}\right)$, since the difference is of second order in the Lorentz violating terms.) Since $\omega$ is proportional to $\sqrt{k}$,

$$
\frac{\delta \omega}{\omega}=\frac{1}{2} \frac{\delta k}{k}=\frac{1}{2 k}\left[\left(\frac{\partial^{3} U}{\partial R^{3}}\right)_{R_{0}} \delta R+\left(\frac{\partial^{2}\langle\delta h\rangle}{\partial R^{2}}\right)_{R_{0}}\right] .
$$

Inserting the numerical values given in Tab. 【 we obtain

$$
\begin{aligned}
& \left(\frac{\delta \omega}{\omega}\right)_{\mathrm{H}_{2}}=-0.337 \operatorname{tr}(E)-0.100\left(E_{3}\right)_{l}^{m}, \\
& \left(\frac{\delta \omega}{\omega}\right)_{\mathrm{H}_{2}^{+}}=-0.926 \operatorname{tr}(\tilde{E})-0.254\left(\tilde{E}_{3}\right)_{l}^{m} .
\end{aligned}
$$

The above treatement of the vibrational transitions is based on a harmonic approximation for the core-core potential near equilibrium. This approximation is relatively accurate for low excitations. For example, the energy of the $v=4$ vibrational level of $\mathrm{H}_{2}^{+}$within the harmonic approximation differs from the realistic value by about $0.6 \%$. Thus, for the vibrational transitions used in the experiments to be discussed below, the harmonic approximation is sufficient.

\section{Rotational transitions}

The rotation of the molecule without Lorentz violation is characterized by an energy $H_{\text {rot }}=1 /\left(2 \bar{m} R_{0}^{2}\right) \vec{L}^{2}$, where $\vec{L}$ is the angular momentum operator with eigenvalues $l(l+1)(l=0,1,2,3, \ldots)$. Transitions between the rotational energy levels thus have a frequency of

$$
\omega_{\text {rot }}\left(l \rightarrow l^{\prime}\right)=\frac{1}{2 \hbar \bar{m} R_{0}^{2}}\left(\frac{1}{l(l+1)}-\frac{1}{l^{\prime}\left(l^{\prime}+1\right)}\right)
$$

Due to Lorentz violation, $R \rightarrow R_{0}+\delta R$; thus, $H_{\text {rot }} \rightarrow$ $H_{\text {rot }}+\delta H_{\text {rot }}$, so the expectation value for the relative shift of the energy levels is

$$
\frac{\delta \omega_{\mathrm{rot}}\left(l \rightarrow l^{\prime}\right)}{\omega_{\mathrm{rot}}\left(l \rightarrow l^{\prime}\right)}=\frac{\left\langle\delta H_{\mathrm{rot}}\right\rangle}{H_{\mathrm{rot}}}=-2 \frac{\delta R}{R_{0}},
$$

where $\delta R / R_{0}$ is given by Eq. (27).

\section{E. Changes due to $\operatorname{tr}(E)$}

The rotation invariant coefficient $\operatorname{tr}(E)$ contained in the Lorentz-violating $\delta h$ Eq. (11) can be absorbed into the conventional Hamiltonian by scaling the mass of the electron:

$$
m_{e} \rightarrow m_{e}\left(1-\frac{2}{3} \operatorname{tr}(E)\right)
$$

This leads to a corresponding scaling of the Bohr radius $a_{0}=4 \pi \epsilon_{0} \hbar^{2} /\left(m_{e} e^{2}\right)$ and the Rydberg constant $R_{\infty}=$

$$
\begin{aligned}
& \alpha^{2} m_{e} c /(2 h): \\
& a_{0} \rightarrow a_{0}\left(1+\frac{2}{3} \operatorname{tr}(E)\right), \quad R_{\infty} \rightarrow R_{\infty}\left(1-\frac{2}{3} \operatorname{tr}(E)\right) .
\end{aligned}
$$

We expect that quantities of dimension energy will scale like the Rydberg constant; quantities of the dimension length should scale like the Bohr radius. In fact, the coefficients of $\operatorname{tr}(E)$ in the change of the ground state energy are close to $-2 / 3$, while those in the length change are close to $+2 / 3$.

The anisotropic effects due to $E_{3}$ can, however, not be predicted by such simple arguments.

\section{TESTS OF LORENTZ INVARIANCE USING MOLECULES}

Experiments using molecules may provide interesting new bounds on some of the Lorentz-violating SME parameters that enter the Hamiltonian, Eq. (II). Present bounds on the SME tensor $c_{\mu \nu}$ are of the order of $10^{-14}$ for the parameter combinations $c_{X Y}$ and $c_{X X}-c_{Y Y}$, and $10^{-12}$ for $c_{X X}+c_{Y Y}-2 c_{Z Z}$. They were derived [11] from experiments [7, 8] based on optical cavities.

Limits on the Lorentz tensors $b_{\mu}, d_{\mu \nu}, g_{\lambda \mu \nu}$, and $H_{\mu \nu}$ from present experiments (e.g., clock-comparison experiments [3]) are sufficiently stringent so that we can neglect these quantities. The reason is that these quantities encode spin-dependent effects, that can be measured to extremely high resolution by monitoring the frequencies of transitions between Zeeman or Hyperfine levels in atoms. For example, from such clock-comparison experiments, $B_{j}^{\prime} \lesssim 10^{-24}[\underline{3}]\left(m B_{j}^{\prime}\right.$ is denoted by $\tilde{b}_{J}$ in the literature); from experiments with spin-polarized solids, components of $B_{j}^{\prime}$ are bounded to a few parts in $10^{-25}[5]$.

Future experiments on Earth and in Space 23] are expected to improve the accuracy of many of the spindependent terms and also [11, 24] of the components of $c_{\mu \nu}$.

To surpass the present limits on the spin-dependent terms in experiments using molecules, some transition between states having different spins would have to be probed with about $10 \mu \mathrm{Hz}$ resolution. This is well below the accuracy that is possible for hydrogen molecules, so we can assume that all Lorentz violating quantities except $c_{\mu \nu}$ are zero and concentrate on setting bounds on $c_{\mu \nu}$.

\section{A. Generic experiment}

If the axis of a molecule (or the axes of an ensemble of molecules) would be aligned and then rotated (e.g., by fixing the axis in the laboratory, which is subject to Earth's rotation and orbital motion), $E_{3}(\theta, \phi)$ will become time dependent. Thus, the basic principle of Lorentz symmetry tests using molecules is to measure a 
property of the molecule, looking for any changes caused by a rotation of the molecule in space. Since frequencies are the quantities in physics that can be measured to highest resolution, the most promising of such experiments are measurements of transition frequencies using laser spectroscopy methods.

For simplicity, we will assume that the molecular axis is fixed in the laboratory. If, instead, the molecule is in an angular momentum eigenstate, the sensitivity of the experiment gets modified according to Eq. (23). Either way, however, a method is needed to fix the quantization axis. There is now a broad range of techniques for aligning molecules (see 27] for a recent review), e.g., using a strong electrostatic field, a hexapole electrostatic field, or polarized laser fields. Most methods need a nonzero electric dipole moment of the molecule. The dipole moment of $\mathrm{HD}$ and $\mathrm{HD}^{+}$is nonzero, but small $\left(\sim 5.8 \times 10^{-4} \mathrm{D}\right.$ [26] for HD). Another method of aligning the molecules, based on pulsed laser fields, uses the anisotropic dipole polarizability and hence also works for molecules having zero dipole moment. A detailed discussion of these techniques is beyond the present scope and can be found in the literature [27].

\section{B. Possible experiments}

Experiments may use (i) transitions between different electronic energy levels or (ii) (ro-) vibrational transitions within one electronic state, preferably the ground state.

\section{Electronic transitions}

Electronic transitions in hydrogen moelcules are difficult to measure to extremely high resolution because of the natural linewidth of those transitions, limited by the lifetime of the excited electronic states. Moreover, the transition frequencies generally lie within the uv region of the spectrum, where narrow-band laser radiation is difficult to generate. For example, the transition between the ground state $X^{1} \Sigma_{g}^{+}$and the excited state $E F^{1} \Sigma_{g}^{+}$of neutral $\mathrm{H}_{2}$ is at a wavelength of about $\lambda=80 \mathrm{~nm}$ (corresponding to a frequency of $\sim 3 \times 10^{15} \mathrm{~Hz}$ ). A combination of frequency multiplied $\sim 10 \mathrm{~ns}$ pulsed and continuous wave lasers can be used for spectroscopy [22], which results in linewidths of several tens of $\mathrm{MHz}$, or $\sim 3 \times 10^{-9}$ of the transition frequency.

The signal for Lorentz violation would be the differential shift of the ground state energy, Eq. (24), and the energy of the excited electronic state, which has to be calculated separately. Such a calculation can also be based on the Born-Oppenheimer method, starting from a linear combination of atomic orbitals like Eq. (5) where one or both of the orbitals are excited states of the hydrogen atom. The resulting differential energy shift will be in analogy to Eq. (24), with adjusted numerical coefficients. To improve the present limits on $c_{\mu \nu}$, a electronic transition needs to be probed to a resolution of about $10^{-15}$, wich appears unlikely to be reached in the near future. Thus, it is uneccesary to work out those theoretical details here.

\section{2. (Ro-) vibrational transitions}

For $\mathrm{H}_{2}$ and $\mathrm{H}_{2}^{+}$, the rotational and vibrational transitions within one electronic level are dipole-forbidden due to the symmetry of the molecules. They are allowed for $\mathrm{HD}$ and $\mathrm{HD}^{+}$, however.

An experiment using $\mathrm{HD}^{+}$is currently in preparation [12, 13. Ionized molecules are used, because they can be cooled using sympathetic cooling by laser cooled atomic ions, eliminating the Doppler broadening of the transitions that occurs at room temperature as a consequence of the thermal motion of molecules in the gas phase. For the spectroscopy in this experiment, a wavelength of $\lambda=1.4 \mu \mathrm{m}$ may be used (among other possibilities), which would drive transitions between rotational sublevels of the $v=1$ and $v=4$ vibrational levels, see Fig. 1 in [13]; continuous wave laser radiation with excellent spectral properties can be generated at this wavelength. The linewidth is thus expected to be limited by the natural linewidth, of the order of tens of $\mathrm{Hz}$ for the dipoleallowed vibrational transitions in the electronic ground state (and below $1 \mathrm{~Hz}$ for pure rotational transitions in the lowest vibrational state) [13]. The signal for Lorentz violation for this experiment is given by Eq. (30) 25]; its time dependence is treated below.

The basic advantage of such experiments with molecules rather than cavities is the lower linewidth of the (ro-) vibrational transitions: While a given relative change of the resonance frequency of an optical cavity 11] and a ro-vibrational transition in hydrogen translate into approximately equal estimates for the Lorentzviolating quantities, optical cavities presently used have linewidths of tens of $\mathrm{kHz}$ [7, 9]; the above linewidths of $\mathrm{HD}^{+}$are three orders of magnitude lower. Thus, molecular experiments have a potentially higher resolution.

\section{Time-dependence of the Signal for Lorentz violation}

Since the quantity $E_{3}$ transforms in a nontrivial way under Lorentz boosts and rotations, Lorentz violation will lead to a characteristic time dependence of transition frequencies in molecules ('signal'). The explicit time-dependence of $E_{3}$ is calculated in the appendix. To give an example, consider an experiment based on rovibrational transitions as described above, where the axes of an ensemble of molecules would be oriented horizontally, pointing south. The signal can then be obtained from Eq. A.4 by inserting $\vartheta=0$ :

$$
\frac{\delta \omega}{\omega}=3 \alpha\left[c_{(Y Z)} \sin 2 \chi \sin \omega_{\oplus} T_{\oplus}\right.
$$




$$
\begin{aligned}
& -c_{(X Y)} \cos ^{2} \chi \sin 2 \omega_{\oplus} T_{\oplus}+c_{(X Z)} \sin 2 \chi \cos \omega_{\oplus} T_{\oplus} \\
& \left.-\frac{1}{2}\left(c_{X X}-c_{Y Y}\right) \cos ^{2} \chi \cos 2 \omega_{\oplus} T_{\oplus}\right]
\end{aligned}
$$

where $\chi$ is the geographical colatitude at which the experiment is performed and $\omega_{\oplus} \simeq 2 \pi / 23 \mathrm{~h} 56 \mathrm{~min}$ is the sidereal angular frequency of Earth's rotation. $\alpha$ is the numerical factor of $E_{3}$ in the applicable expression for the Lorentz-violating shift in the quantity measured, i.e., $\alpha=-0.254$ for a vibrational transition in $\mathrm{H}_{2}^{+}$, as given by Eq. (30). Thus, if a resolution of $10^{-16 \ldots-15}$ in the measurement of $\delta \omega / \omega$ is achieved, individual bounds on the Lorentz-violating coefficients $c_{(Y Z)}, c_{(X Z)}, c_{(Y Z)}$, and $c_{X X}-c_{Y Y}$ of about $10^{-15}$ can be expected. This compares favourably with present cavity experiments $[6,7,[8,9,10,11]$ and is competitive with future space projects [24].

In analogy to cavity experiments, the optimum sensitivity would probably be achieved by rotating the setup on a turntable at $\omega_{t}$. The signal, given in the appendix, then consists of Fourier components around $2 \omega_{t}$. While such an experiment requires considerably more effort compared to a setup without turntable, it is sensitive to the additional combination $c_{X X}+c_{Y Y}-2 c_{Z Z}$ of Lorentz violating coefficients. Moreover, $\omega_{t}$ can be chosen to match the time scale of the optimum sensitivity of the experiment, which could be much lower than $12 \mathrm{~h}$. In this case, not only the accuracy of the data acquired during one rotation will be better, but averaging over many rotations will also lead to improved statistics in the same time of measurement.

\section{DISCUSSION, SUMMARY AND OUTLOOK}

We investigated the properties of the neutral and ionized hydrogen molecule $\mathrm{H}_{2}$ and $\mathrm{H}_{2}^{+}$under the influence of Lorentz violation in the electrons' equation of motion. We find Lorentz-violating changes of the frequencies of electronic and (ro-) vibrational transitions, and of the bond length. The latter can be compared to the geometry change of solids calculated in [1]. The calculations for molecules and solids use dissimilar models and approximations. The agreement of the results (in both cases the Lorentz-violating relative length change is given by the elements of $E$ and $\tilde{E}$ together with numerical coefficients of the order of one) is thus interesting and confirms the reliability of both calculations.

The sensitivity of the molecules' properties on the parameter combinations $\operatorname{tr}(E)$ and $E_{3}$ allows to derive upper limits on Lorentz violation: Precision calculations and measurements of the ground state energy of $\mathrm{H}_{2}$ agree to about $1 \times 10^{-7}$, which allows us to place limits of this order of magnitude on $\operatorname{tr}(E)$ and $E_{3}$.

Precision measurements using molecules, e.g., by high resolution spectroscopy of ro-vibrational energy levels within the electronic ground state sympathetically cooled $\mathrm{HD}^{+}$, may lead to individual bounds on the Lorentz- violating coefficients $c_{(Y Z)}, c_{(X Z)}, c_{(Y Z)}$, and $c_{X X}-c_{Y Y}$ of the order of $10^{-15}$ or better. This would compare favorably to the $10^{-14}$ accuracy of the best present cavity experiments and be competitive to future space tests [24]. Experiments using a turntable will additionally measure the parameter combination $c_{X X}+c_{Y Y}-2 c_{Z Z}$ and should yield a further improvement in resolution by at least one order of magnitude.

The theory presented here does not include Lorentz violation in the photon sector, but it can be extended to include it. This would open up the possibility of additional tests of Lorentz symmetry using molecules. The issue of the independent measurability of the electron and photon parameters is discussed in [11, 28].

\section{Acknowledgments}

We would like to thank H. Dehnen, who suggested to conduct this research to us. H.M. acknowledges a FeodorLynen fellowship by the Alexander von HumboldtStiftung. A.S. acknowledges financial support by the Stifterverband für die Deutsche Wissenschaft and the Fonds der chemischen Industrie. C.L. thanks the german space agency DLR for financial support.

\section{APPENDIX: EXPLICIT TIME-DEPENDENCE OF THE SIGNAL}

Here, we give in detail the full signal caused by Lorentz violation in the electrons' equation of motion. We assume that the axis of the molecule is fixed horizontally and then rotated, using a turntable at an angular frequency $\omega_{t}$ (as measured in the laboratory on Earth). The rotation axis is fixed to point vertically. We use a turntable time scale $t_{t}$ defined such that $t_{t}=0$ at any one instant when the cavity is pointing in the $x$ direction of the laboratory frame that is defined below. The signal for experiments where the molecule has a fixed horizontal orientation that has an angle $\theta$ with the $x$ axis can be obtained by setting $\omega_{t} t_{t}=\theta$, see below.

Limits on the Lorentz-violating quantities of the SME are usually expressed within a sun-centered celestial equatorial reference frame. As defined in [3], it has the $X$ axis pointing towards the vernal equinox (spring point) at $0 \mathrm{~h}$ right ascension and $0^{\circ}$ declination, the $Z$ axis pointing towards the celestial north pole $\left(90^{\circ}\right.$ declination) and the $Y$ axis such as to complete the right handed orthogonal dreibein. Earth's equatorial plane lies in the $X-Y$ plane; its orbital plane is tilted by $\eta \simeq 23^{\circ}$ with respect to the latter. The time scale $T=0$ when the sun passes the spring point, e.g., on march 20, 2001 at 13:31 UT.

We also define a laboratory frame, which has the $x$ axis pointing south, the $y$ axis east, and the $z$ axis vertically upwards. The laboratory time scale $T_{\oplus}=0$ when the $y$ and the $Y$ axis coincide. 
The signal derivation starts from the symmetrized tensor $c_{(\mu \nu)}$ given in the sun-centered celestial equatorial reference frame. The first step would be a Lorentz boost according to $\beta_{\oplus} \simeq 10^{-4}$, the velocity of Earth's orbit. However, because of the symmetry of the molecule, this turns out to generate no first order contributions to the signal. Taking into account also the second order is unnecessary because it is suppressed by a factor $\beta_{\oplus}^{2} \sim 10^{-8}$.

Application of the rotation matrix

$$
R=\left(\begin{array}{ccc}
\cos \chi \cos \omega_{\oplus} T_{\oplus} & \cos \chi \sin \omega_{\oplus} T_{\oplus} & -\sin \chi \\
-\sin \omega_{\oplus} T_{\oplus} & \cos \omega_{\oplus} T_{\oplus} & 0 \\
\sin \chi \cos \omega_{\oplus} T_{\oplus} & \sin \chi \sin \omega_{\oplus} T_{\oplus} & \cos \chi
\end{array}\right)
$$

where $\chi$ is the geographical colatitude, and $\omega_{\oplus} \simeq$ $2 \pi / 23 \mathrm{~h} 56 \mathrm{~min}$ Earth's rotation angular frequency, leads to the tensor $c_{\mu \nu}$ as expressed within the laboratory frame. Another rotation around the $z$ axis using the rotation matrix

$$
R_{t}=\left(\begin{array}{ccc}
\cos \omega_{t} t_{t} & \sin \omega_{t} t_{t} & 0 \\
-\sin \omega_{t} t_{t} & \cos \omega_{t} t_{t} & 0 \\
0 & 0 & 1
\end{array}\right)
$$

leads to the quantities within the rotating turntable frame, which are then decomposed according to Eq. (3). A last rotation by a right angle aligns the molecules $z$ axis to the $x_{t}$ axis.

The properties of hydrogen molecules depend on the parameter combinations $\operatorname{tr} E$ and $E_{3}=\operatorname{tr} E-3 E_{z z}$. Because of the rotation invariance of the trace, only $E_{3}$ becomes time dependent. $E_{3}$ can be expressed as a Fourier series

$$
\begin{aligned}
E_{3}= & C(0,0)+\sum_{a, b}\left[S(a, b) \sin \left(a \omega_{t} t_{t}+b \omega_{\oplus} T_{\oplus}\right)\right. \\
& \left.+C(a, b) \cos \left(a \omega_{t} t_{t}+b \omega_{\oplus} T_{\oplus}\right)\right]
\end{aligned}
$$

with coefficients $S(a, b)$ and $C(a, b)$; the dc component $C(0,0)$ is not included in the equations below, as it is not measurable. We have seven signal frequencies with the amplitudes

$$
\begin{aligned}
& C(0,1)=3 c_{(X Z)} \cos \chi \sin \chi, \\
& S(0,1)=3 c_{(Y Z)} \cos \chi \sin \chi, \\
& C(0,2)=\frac{3}{4}\left(c_{X X}-c_{Y Y}\right) \sin ^{2} \chi, \\
& S(0,2)=\frac{3}{2} c_{(X Y)} \sin ^{2} \chi, \\
& C(2,-2)=-\frac{3}{2}\left(c_{X X}-c_{Y Y}\right) \sin ^{4} \frac{\chi}{2}, \\
& S(2,-2)=3 c_{(X Y)} \sin ^{4} \frac{\chi}{2}, \\
& C(2,-1)=-6 c_{(X Z)} \cos \frac{\chi}{2} \sin ^{3} \frac{\chi}{2}, \\
& S(2,-1)=6 c_{(Y Z)} \cos \frac{\chi}{2} \sin ^{3} \frac{\chi}{2}, \\
& C(2,0)=\frac{3}{4}\left(c_{X X}+c_{Y Y}-2 c_{Z Z}\right) \sin ^{2} \chi, \\
& S(2,0)=0 \text {, } \\
& C(2,1)=6 c_{(X Z)} \cos ^{3} \frac{\chi}{2} \sin \frac{\chi}{2}, \\
& S(2,1)=6 c_{(Y Z)} \cos ^{3} \frac{\chi}{2} \sin \frac{\chi}{2}, \\
& C(2,2)=-\frac{3}{2}\left(c_{X X}-c_{Y Y}\right) \cos ^{4} \frac{\chi}{2}, \\
& S(2,2)=-3 c_{(X Y)} \cos ^{4} \frac{\chi}{2} \text {. }
\end{aligned}
$$

The time dependence for experiments without turntable can be obtained from these coefficients by letting $\omega_{t} t_{t}=$ $\vartheta$, where $\vartheta$ is the angle of the (horizontally oriented) molecular axis with respect to the north-south axis. We obtain

$$
\begin{aligned}
E_{3}= & \sum_{a, b}[S(a, b) \cos a \vartheta-C(a, b) \sin a \vartheta] \sin b \omega_{\oplus} T_{\oplus} \\
& +[S(a, b) \sin a \vartheta+C(a, b) \cos a \vartheta] \cos b \omega_{\oplus} T_{\oplus} \cdot(\mathrm{A} .4)
\end{aligned}
$$

[1] D. Colladay and V.A. Kostelecký, Phys. Rev. D 55, 6760 (1997); ibid. 58, 116002 (1998).

[2] V.A. Kostelecký, Phys. Rev. D 69, 105009 (2004); V.A. Kostelecký and Matthew Mewes, Phys. Rev. Lett. 87, 251304 (2001); Phys. Rev. D 66, 056005 (2002); ibid. 69, 016005 (2004).

[3] V.A. Kostelecký and C. D. Lane, Phys. Rev. D 60, 116010 (1999).

[4] R. Bluhm, V.A. Kostelecký, and N. Russell, Phys. Rev. Lett. 82, 2254 (1999).

[5] R. Bluhm and V.A. Kostelecký, Phys. Rev. Lett. 84, 1381 (2000); E.G. Adelberger et al. in: Physics beyond the Standard Model, edited by P. Herszeg et al., World Scientific, Singapore (1999); L.-S. Hou, W.-T. Ni, and Yu-ChuM. Li, Phys. Rev. Lett. 90, 201101 (2003).
[6] A. Brillet and J.L. Hall, Phys. Rev. Lett. 42, 549 (1979).

[7] H. Müller et al., Phys. Rev. Lett. 91, 020401 (2003); Appl. Phys. B (laser opt.) 77, 719 (2003); Opt. Lett. 28, 2186 (2003).

[8] P. Wolf et al., Phys. Rev. Lett. 90, 060402 (2003); Phys. Rev. D, Rapid Communications, in press (hep-ph/0407232); J. Lipa et al., Phys. Rev. Lett. 90, 060403 (2003).

[9] C. Braxmaier et al., Phys. Rev. Lett. 88, 010401 (2001); H. Müller et al., Int. J. Mod. Phys. D 11, 1101 (2002).

[10] H. Müller et al., Phys. Rev. D 67, 056006 (2003).

[11] H. Müller et al., Phys. Rev. D 68, 116006 (2003).

[12] S. Schiller and C. Lämmerzahl, Phys. Rev. A 68, 053406 (2003).

[13] U. Fröhlich, B. Roth, P. Antonini, C. Lämmerzahl, A. 
Wicht, and S. Schiller, to appear in: Proc. of the WEHeraeus Seminar on Astrophysics, Clocks and Fundamental Constants (2004).

[14] S. Flügge, Practical Quantum Mechanics (Springer, Berlin, Germany, 1994).

[15] C. Lämmerzahl, Class. Quantum Grav. 15, 13 (1998).

[16] V.A. Kostelecký and C.D. Lane, J. Math. Phys. 40, 6245 (1999).

[17] We note misprints in Ref. [14]: On p. 86, two signs have been reversed in the equation giving $\varphi(\rho)$ such that the energy of $\mathrm{H}_{2}$ would go to $-\infty$ as $R \rightarrow 0$. The correct expression reads $\varphi(\rho)=S(\rho)^{2}(\log \rho+C)+S(-\rho)^{2} E_{1}(4 \rho)-$ $2 S(\rho) S(-\rho) E_{1}(2 \rho)$.

[18] L.D. Landau, E.M. Lifschitz, Quantenmechanik, (Akademie-Verlag, Berlin, Germany, 1979), § 107.

[19] Here, we used $\left\langle p_{x}^{2}\right\rangle E_{x x}+\left\langle p_{y}^{2}\right\rangle E_{y y}+\left\langle p_{z}^{2}\right\rangle E_{z z}=$ $\left(\frac{2}{3}\left\langle p_{x}^{2}\right\rangle+\frac{1}{3}\left\langle p_{z}^{2}\right\rangle\right) \operatorname{tr}(E)+\frac{1}{3}\left(\left\langle p_{x}^{2}\right\rangle-\left\langle p_{z}^{2}\right\rangle\right) E_{3}$

[20] M. Caifero, S. Bubin, and L. Adamowicz, Phys. Chem. Chem. Phys. 5, 1491 (2003); C.A. Leach and R.E. Moss, Ann. Rev. Phys. Chem. 46, 55-82 (1995); W. Kolos, J. Chem. Phys. 101, 1330 (1994); L. Wolniewicz, J. Chem.
Phys. 103, 1792 (1995); ibid. 99, 1851 (1993); M.M. Cassar and G.W.F. Drake, J. Phys. B 37, 2485 (2004).

[21] D. Shiner et al., Phys. Rev. A 47, 4042 (1993); A. de Lange, E. Reinhold, and W. Ubachs, Phys. Rev. A 65, 064501 (2002); W. Ubachs and E. Reinhold, Phys. Rev. Lett. 92, 101302 (2004).

[22] E. McCormack et al., Phys. Rev. A 39, 2260 (1989); J.M. Gilligan and E.E Eyler, ibid. 46, 3676 (1992).

[23] R. Bluhm et al., Phys. Rev. Lett. 88, 090801 (2002); Phys. Rev. D 68, 125008 (2003); C. Lämmerzahl et al., Class. Quantum Grav. 18, 2499 (2001).

[24] C. Lämmerzahl et al., Gen. Rel. Grav. 36, 2373 (2004).

[25] Plus a small contribution of the energy shifts in the rotational sublevels.

[26] M. Trefler and H.P. Gush, Phys. Rev. Lett. 20, 703 (1968).

[27] For a recent review, see H. Stapelfeld and T. Seideman, Rev. Mod. Phys. 75, 543 (2003).

[28] Q.G. Bailey and V.A. Kostelecký, hep-ph/0407252 (2004). 